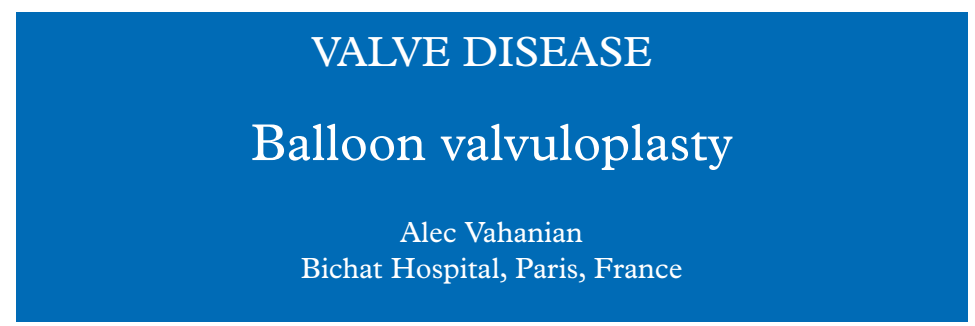

Thtil the early 1980s surgery was the only possible treatment for severe valvar stenosis. Then a new alternative appeared-percutaneous balloon valvuloplasty.

I will deal here with percutaneous valvuloplasty for acquired valvar stenoses in the fields of mitral stenosis, aortic stenosis, and, although it occurs far less frequently, tricuspid or bioprosthetic stenoses.

\section{Percutaneous mitral commissurotomy}

Rheumatic mitral stenosis continues to be endemic in developing countries where mitral stenosis is the most frequent valve disease. ${ }^{1}$ Although the prevalence of rheumatic fever has greatly decreased in western countries, it continues to represent an important clinical entity because of immigration from developing countries.

The first to perform percutaneous mitral commissurotomy (PMC) as an alternative to surgery was Inoue in $1982 .^{2}$ The good results obtained with the technique have led to its increasing worldwide use and its positioning as the second most important technique in the field of interventional cardiology.

\section{Evaluation before PMC}

Clinical evaluation is the first step when deciding whether to operate or intervene. Under particular scrutiny here are functional disability and any possible risks with surgery.

The assessment of anatomy aims to eliminate contraindications and define prognostic considerations. Echographic assessment allows the classification of patients into anatomic groups with a view to predicting the results. Most authors use the Wilkins score (table 1) while others, like Cormier (table 2), use a more general assessment of valve anatomy. ${ }^{3}$ More recently, scores which take into account the uneven distribution of anatomic abnormalities, particularly in regard to commissural areas, have been developed. In fact, none of the scores available has been shown to be superior to any of the others, and we can only recommend the use of the score with which one is most familiar and at ease.

Correspondence to: Professor Alec Vahanian, Groupe Hospitalier Bichat-Claude Bernard, 46, rue Henri-Huchard, 75877 Paris Cedex 18, France alec.vahanian@bch. ap-hop-paris.fr
Table 1 Anatomic classification of the mitral valve (Wilkins'score, Massachusetts General Hospital)

Leaflet mobility

- Highly mobile valve with restriction of only the leaflet tip

- Mid portion and base of leaflets have reduced mobility

- Valve leaflets move forward in diastole mainly at the base

- No or minimal forward movement of the leaflets in diastole

Valvar thickening

- Leaflets near normal (4-5 mm)

- Mid leaflet thickening, pronounced thickening of the margins

- Thickening extends through the entire leaflets $(5-8 \mathrm{~mm})$

- Pronounced thickening of all leaflet tissue $(>8-10 \mathrm{~mm})$

Subvalvar thickening

- Minimal thickening of chordal structures just below the valve

- Thickening of chordae extending up to one third of chordal length

- Thickening extending to the distal third of the chordae

- Extensive thickening and shortening of all chordae extending down to the papillary muscle

Valvar calcification

- A single area of increased echo brightness

- Scattered areas of brightness confined to leaflet margins

- Brightness extending into the mid portion of leaflets

- Extensive brightness through most of the leaflet tissue

Table 2 Anatomic classification of the mitral valve (Cormier's score, Tenon and Bichat Hospitals, Paris)

\begin{tabular}{ll}
\hline $\begin{array}{l}\text { Echocardiographic } \\
\text { group }\end{array}$ & Mitral valve anatomy \\
\hline Group 1 & $\begin{array}{l}\text { Pliable non-calcified anterior mitral leaflet and } \\
\text { mild subvalvar disease-that is, thin chordae } \\
\geqslant 10 \text { mm long }\end{array}$ \\
Group 2 & $\begin{array}{l}\text { Pliable non-calcified anterior mitral leaflet and } \\
\text { severe subvalvar disease-that is, thickened } \\
\text { chordae }<10 \text { mm long } \\
\text { Calcification of mitral valve of any extent, as } \\
\text { assessed by fluoroscopy, whatever the } \\
\text { subvalvar apparatus }\end{array}$ \\
\hline
\end{tabular}

rare cases where the transeptal approach is contraindicated. ${ }^{4}$

There are currently two main techniquesballoon commissurotomy, and metallic commissurotomy.

\section{Balloon commissurotomy}

In balloon commissurotomy the two major techniques are the double balloon technique and the Inoue technique.

The double balloon technique requires the use of a floating balloon catheter to cross the mitral valve and then the positioning of two guidewires in the apex of the left ventricle. It is effective but demanding, and carries the risk of left ventricular perforation by the guidewires or the tip of the balloons (fig 1). The multi-track system is a recent variant of the double balloon technique and aims to make the procedure easier, as it only requires the presence of a single guide wire (fig 2).

The design of the Inoue balloon allows safe and fast positioning across the valve. In addition, it is pressure extensible, allowing for the performance of a stepwise dilatation (fig 3). The data available comparing the Inoue and the double balloon techniques suggest that the Inoue technique makes the procedure easier and has equivalent efficacy, but with lower risk. In fact the Inoue technique has become the most popular worldwide. 


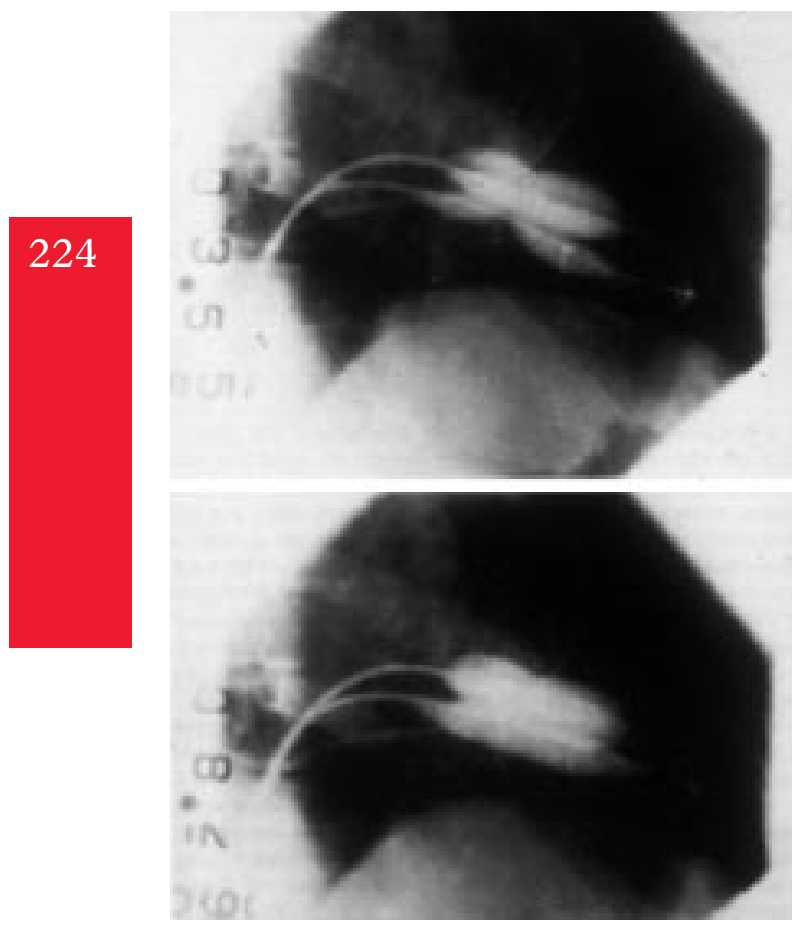

Figure 1. Transvenous technique using the combination of a trefoil and a conventional balloon

\section{Metallic commissurotomy}

Recently Cribier introduced the metallic commissurotomy (fig 4), which uses a device similar to the Tubb's dilator used during closed surgical commissurotomy. ${ }^{5}$ The experience with this device is only preliminary and exclusively reported by the promoter of the method. These initial results suggest that its efficacy is similar to balloon commissurotomy, but the risk of haemopericardium seems higher because of the device and the presence of a guide wire in the left ventricle. In addition, this technique is more demanding for the operator than the Inoue technique. The potential advantage of metallic commissurotomy is that the dilator is reusable, which will reduce the cost of the procedure. This is of interest in developing countries, where high rates of rheumatic disease often co-exist with low financial means, limiting the use of percutaneous dilatation.

The definite comparison of the respective merits of the two methods requires further data concerning metallic commissurotomy and randomised comparisons of the two techniques.

The results of PMC in the catheterisation laboratory can be assessed haemodynamically or by echocardiography. Although echocardiography may be difficult to perform in the catheterisation laboratory for logistical reasons, it is very important as it enables the detection of early complications and provides essential information on the course of the mitral opening; this is crucial when using a stepwise Inoue technique. The following criteria have been proposed for the desired end point of the procedure: valve area $>1 \mathrm{~cm}^{2} / \mathrm{m}^{2}$ body surface area (BSA); complete opening of at least one commissure; appearance or increment of regurgitation greater than grade 1 in the Sellers 0 to 4 classification. These are of course only
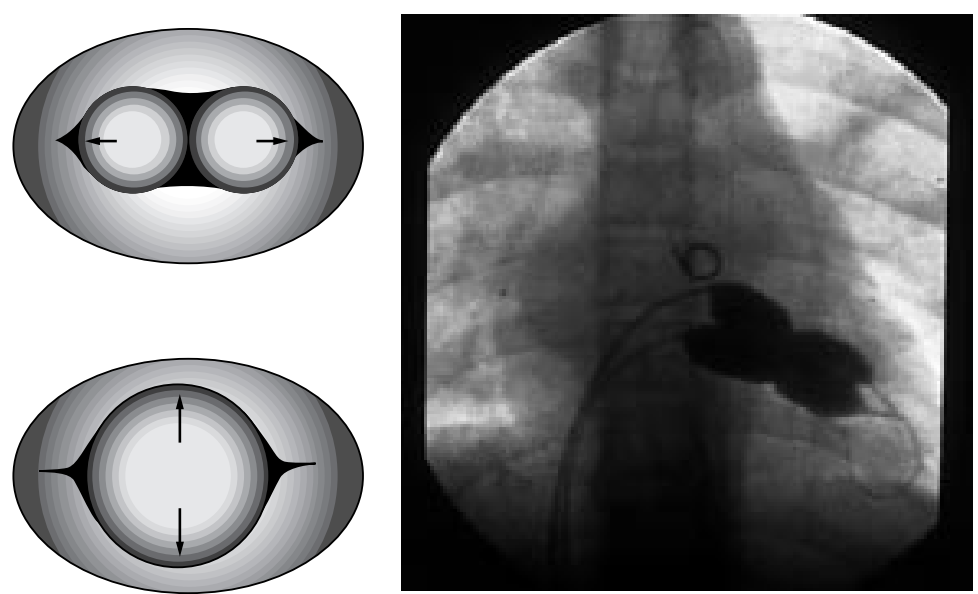

Figure 2. Multi-track technique: inflation of two balloons across the mitral valve-note the presence of one guidewire in the apex. Courtesy of $\operatorname{Dr} \mathrm{P}$ Bonhoeffer.

indications and it is necessary to tailor the strategy according to individual circumstances.

After the procedure, the most accurate evaluation of valve area is provided by echocardiography using planimetry whenever possible. The final assessment of the degree of regurgitation may be made by angiography and by Doppler colour flow. Transoesophageal examination is recommended after the procedure to determine the mechanisms involved in case of severe regurgitation.

\section{Results}

The technique has now been evaluated in several thousand patients with different clinical circumstances. ${ }^{67}$
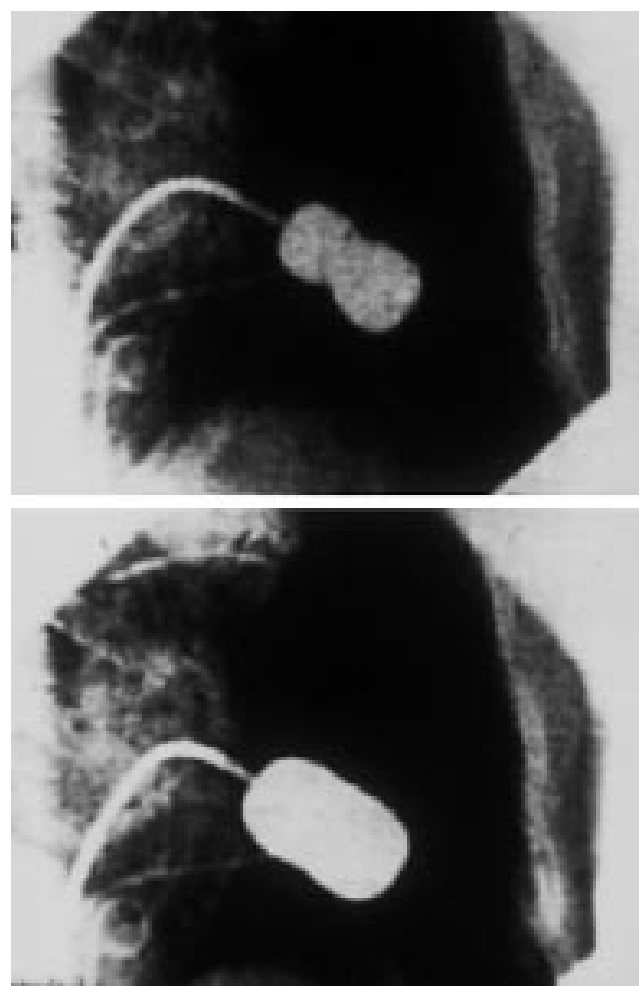

Figure 3. The Inoue balloon technique with the balloon at full inflation. The balloon's "waist" located at its mid-portion disappears. 


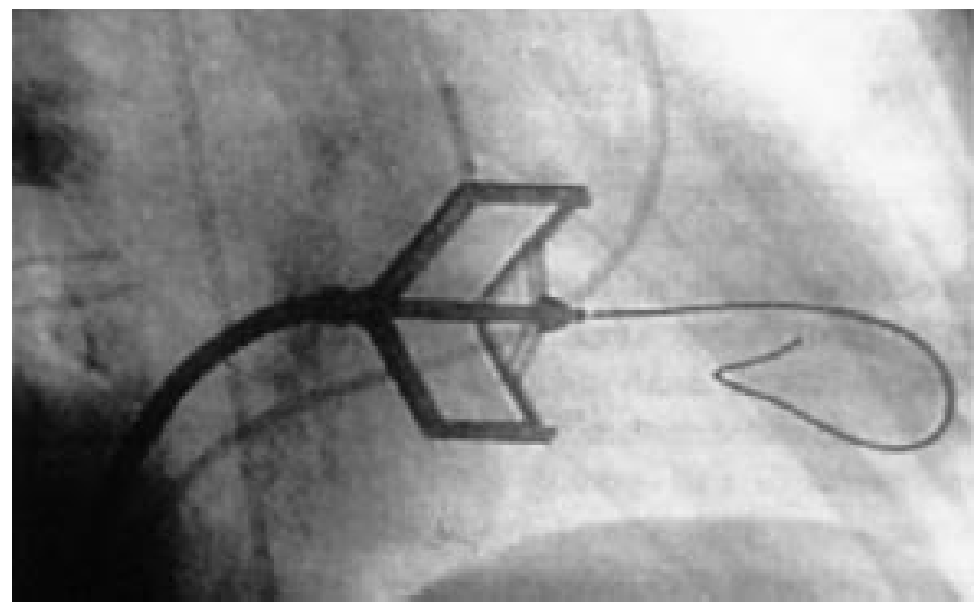

Figure 4. Metallic commissurotomy: opening of the metallic commissurotome across the mitral valve. Courtesy of $\operatorname{Dr}$ A Cribier.

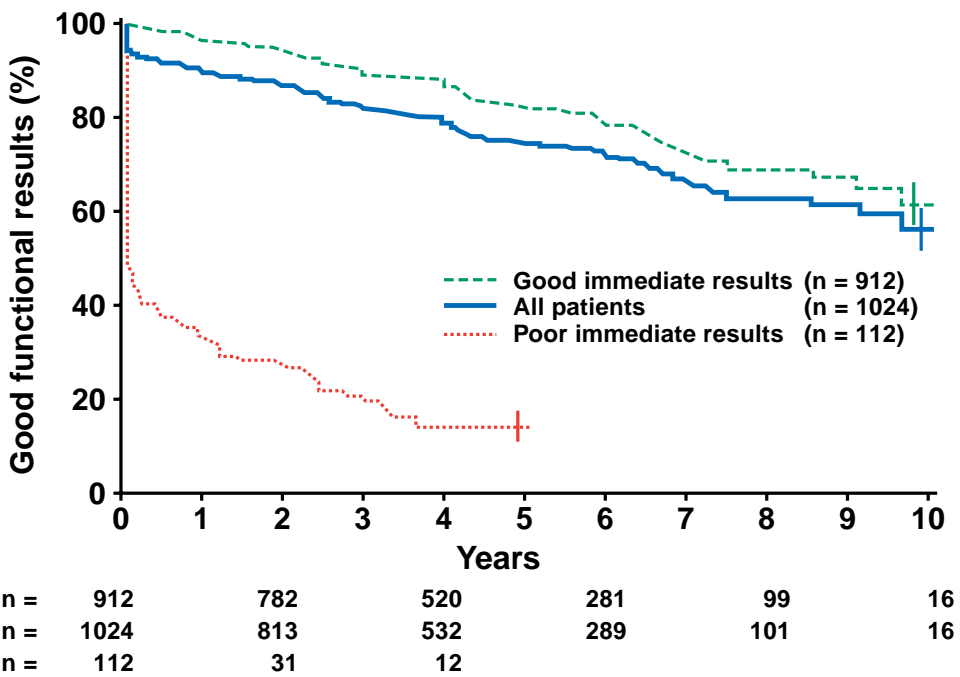

Figure 5. Percutaneous mitral commissurotomy: good functional results (survival considering cardiovascular related deaths with no need for mitral surgery or repeat dilatation and in New York Heart Association functional class I or II). Reproduced with permission from lung B, et al. Circulation 1999;3272-8, with permission of the publisher.

PMC usually provides over $100 \%$ increase in valve area, with a final valve area of $2 \mathrm{~cm}^{2}$ on average. The improvement in valve function results in an immediate decrease in left atrial and pulmonary pressures, both at rest and on exercise.

Risks

The failure rates range from $1-15 \%$ and they mostly occur soon after the procedure, in the investigators' experience. Procedural mortality ranges from $0-3 \%$. The incidence of haemopericardium varies from $0.5-12 \%$, and embolism is encountered in $0.5-5 \%$ of cases. Severe mitral regurgitation is the most worrying complication. ${ }^{8}$ It occurs in $2-10 \%$ of patients, and results from non-commissural leaflet tearing, mostly in cases with unfavourable anatomy, and even more so if there is a heterogeneous distribution of the morphologic abnormalities. Surgery is often necessary later and can be conservative in cases with less severe valve deformity. Although urgent surgery (within 24 hours) is seldom needed for complications, it may be required for massive haemopericar- dium intractable by pericardiocentesis or, less frequently, for severe mitral regurgitation, leading to haemodynamic collapse or refractory pulmonary oedema. Immediately after PMC colour Doppler echo shows small intra-atrial shunts in $40-80 \%$ of cases. However, oximetry shows that the QP:QS ratio is seldom over 1.5 .

The complication rate of the procedure is clearly related to the experience of the team. PMC should probably be restricted to groups whose experience of transseptal catheterisation has been positive and who have been able to carry out an adequate number of procedures, thus improving their technical performance and ability to select patients. This recommendation carries even more weight in western countries, where mitral stenosis is infrequent.

\section{Predictors of immediate results}

The prediction of the immediate results is multifactorial. Besides morphological factors, pre-operative variables such as age, history of commissurotomy, functional class, small mitral valve area, presence of tricuspid regurgitation, as well as procedural factors such as the non-use of the Inoue technique, are all independent predictors of poor immediate results.

\section{Long term results}

We are now able to analyse follow up data up to 10 years. In clinical terms, several large single centre series confirm the late efficacy of PMC in a large population comprising a variety of patient subsets (fig 5). ${ }^{9}$ As shown with surgical commissurotomy, late outcome after PMC differs according to the quality of the immediate results.

When the immediate results are unsatisfactory, patients experience only transient or no functional improvement. The prognosis for patients with severe mitral regurgitation is usually poor and surgical treatment is usually required in the months following. In cases of insufficient initial opening, delayed surgery is usually performed when the clinical conditions allow it. However, in some patients moderate improvement in valve function provides functional improvement for several years, although they must be carefully followed to allow for a timely operation.

Conversely, if PMC is initially successful survival rates are excellent, the need for subsequent surgery is infrequent, and functional improvement occurs in the majority of cases. When functional deterioration occurs in these patients it is late and mainly related to mitral restenosis. The incidence of restenosis is around $40 \%$ after seven years. ${ }^{10}$ The possibility of repeating PMC in cases of recurrent mitral stenosis is one of the potentials of this non-surgical procedure. Repeat PMC can be proposed if recurrent stenosis leads to symptoms, occurs several years after an initially successful procedure, and if the predominant mechanism of restenosis is commissural refusion. At the moment, we have available only a very small number of series on re-PMC, showing encouraging results in selected patients, but 
Contraindications for percutaneous mitral commissurotomy

Left atrial thrombosis

Mitral regurgitation $>2 / 4$

- Massive or bicommissural calcification

- Severe aortic valve disease, or severe tricuspid stenosis + regurgitation, associated with mitral stenosis

Severe concomitant coronary artery disease requiring bypass surgery

the exact role of re-PMC can only be defined when we have larger series with longer follow up.

Follow up studies using sequential transoesophageal echocardiographic examinations have shown that the degree of mitral regurgitation remains on the whole stable or slightly decreases during follow up. Atrial defects are likely to close later in the majority of cases. Successful PMC decreases the intensity of spontaneous left atrial contrast, reduces the size of the left atrium, and improves left atrial function. Even if these findings do not constitute proof of the efficacy of PMC on thromboembolism or atrial fibrillation, they consistently show the beneficial effect of the procedure on their causes.

\section{Predictors of long term results}

Prediction of the long term results is multifactorial, based on clinical variables such as age, valve anatomy, factors related to the stage of the disease (such as functional class), atrial fibrillation, history of previous commissurotomy, severe tricuspid regurgitation, cardiomegaly, and high pulmonary pressure. ${ }^{910}$ Finally, the long term outcome is closely related to the quality of the immediate results, as assessed by final gradient, valve area, and degree of regurgitation.

\section{Selection of the candidates}

Contraindications to PMC are summarised in the box below. The most important is the presence of left atrial thrombosis which can be detected by the systematic performance of transoesophageal echocardiography a few days before PMC. A contraindication is self-evident if the thrombus is floating, localised in the cavity or on the interatrial septum. However, no consensus has been reached in cases with thrombosis localised in the left atrial appendage. In our opinion, in such cases the indications for PMC should be limited to patients with contraindications to surgery, or those without urgent need for intervention when oral anticoagulation can been given for at least one month before PMC, and a new transoesophageal echocardiographic examination shows the disappearance of the thrombus.

It has been suggested that PMC should be performed in patients with moderate stenosis in the hope of delaying the natural course of the disease. However, these patients are usually candidates for medical treatment, and the risks of PMC outweigh the benefits.

\section{Indications for percutaneous mitral commissurotomy}

PMC is the procedure of choice when surgery is contraindicated ${ }^{11}$ or for patients with favourable characteristics - that is, young patients with favourable anatomy. In this latter population several randomised studies comparing PMC and surgical commissurotomy are now available. ${ }^{12}$ They show that PMC is at least comparable to surgical commissurotomy as regards immediate and long term results, and is no doubt more comfortable for the patient. In addition, if restenosis occurs, these patients could undergo repeat PMC or surgery without the difficulties and inherent risks resulting from pericardial adhesion and chest wall scarring.

On the other hand, much remains to be done in refining indications for the other patients, especially those with minimal symptoms and those with unfavourable anatomy.

In cases of symptomatic patients, the indications for PMC are perfectly clear. Because of the small but definite risk inherent in the technique, however, truly asymptomatic patients are not usually candidates for the procedure, except in the following cases: increased risk of thromboembolism-for example a previous history of embolism, dense spontaneous contrast in the left atrium, or recurrent atrial fibrillation; need for major extracardiac surgery; or finally to allow pregnancy. In such patients, PMC should only be performed by experienced interventionists and if valve anatomy is favourable, in which case a safe and successful procedure can be expected.

Patients with unfavourable anatomy are common in western countries. Unfortunately, no randomised study is available for these patients and a comparison of the results of PMC with those of surgical series is difficult because of the differences in the patients involved. For this group of patients some favour immediate surgery because of the less satisfying results of PMC, whereas others prefer PMC as an initial treatment for selected patients, resorting to surgery in the event of failure. In such cases the decision must be individualised and one should take into account the multifactorial nature of the prediction of the results for patient selection. ${ }^{9}$ Data available suggest that continuing good long term results may be obtained and PMC may be useful to defer surgery in selected patients with mild to moderate calcification or severe impairment of the subvalvar apparatus, but with otherwise favourable characteristics. ${ }^{13}$

The same strategy can also be proposed when the risk of surgery is high-in the elderly ${ }^{11}{ }^{14}$ where PMC can be considered as a palliative treatment, in patients with a previous history of surgical commissurotomy ${ }^{15}$ or aortic valve replacement, and during pregnancy if symptoms persist despite medical treatment. ${ }^{16}$ 


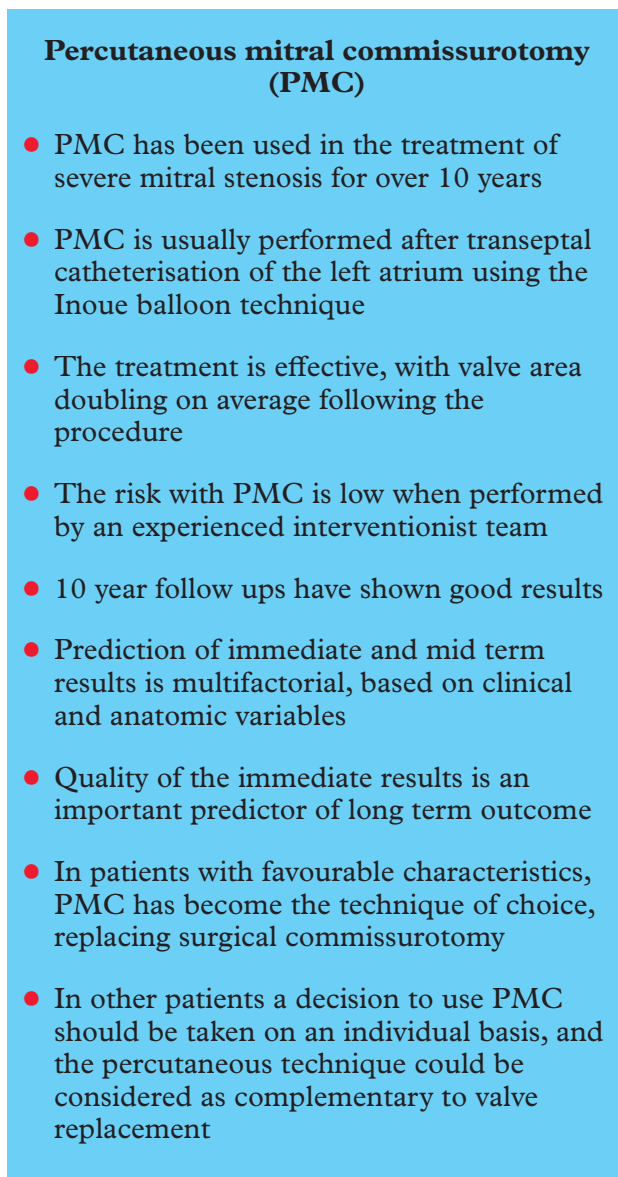

Percutaneous aortic valvuloplasty

Severe degenerative calcified aortic stenosis is the most frequent valve disease in western countries, which accounts for the initial interest in its potential treatment by interventional cardiology. The percutaneous aortic valvuloplasty (PAV) technique was described by Cribier in $1985 .{ }^{17}$

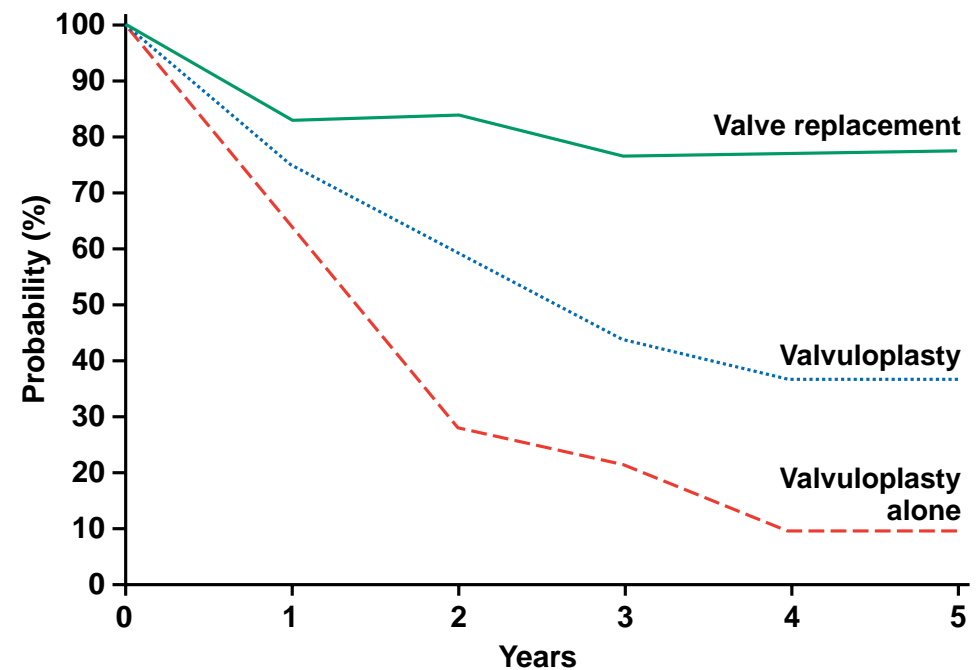

Figure 6. Long term survival rate after aortic valve replacement or balloon valvuloplasty. Reproduced with permission from the American College of Cardiology, J Am Coll Cardiol 1992;20:796-801.
Technique

The femoral approach is the most frequently used. The alternative is the antegrade approach which necessitates a transseptal catheterisation and results in a difficult procedure. Valvuloplasty is performed with balloons from 15$25 \mathrm{~mm}$ in diameter.

The most viable method for assessing the results is measuring the valve area by echo Doppler in the days following the procedure. Aortography before and after the procedure evaluates any changes in the degree of aortic incompetence.

\section{Results}

As could be expected from the anatomic lesions in severe degenerative aortic stenosisthat is, absence of commissural fusion and extensive calcification-PAV has only a limited efficacy. Overall it reduces tight stenosis to moderate stenosis with a final valve area between $0.7-1.1 \mathrm{~cm}^{2}$. This is clearly inferior to the valve area obtained with a valvar prosthesis, which usually provides a valve area over $1.5 \mathrm{~cm}^{2}$.

\section{Risks}

Mortality and morbidity of the procedure are high. Hospital mortality varies from 3.5$13.5 \%$, and within 24 hours $20-25 \%$ of the patients have at least one serious complication, in particular vascular complications at the puncture site. $^{18}$

\section{Long term results}

Despite a relatively modest improvement in valve function, it is common to note a degree of functional improvement during the first months; this functional improvement is, however, of short duration. It seems that PAV improves survival rates at one year and especially the quality of life; beyond one year, however, the benefit decreases and finally disappears after two years. ${ }^{19}$ With selected patients, an aortic valve replacement has been subsequently performed with good results, but on the other hand the prognosis for the other patients is particularly poor (fig 6).$^{20}$ Overall it is now admitted that PAV alone does not change the natural course of the disease. The poor mid-term results are mainly due to the clinical status of the patients and to the moderate and transient improvement in valve function obtained by PAV.

\section{Patient selection}

There are no randomised comparisons available between PAV and surgery. Therefore the indications should take into account the excellent results of aortic valve replacement when it is possible, and the poor results of PAV.

The question today is whether or not there is still really a place for PAV. Most groups have abandoned the technique, while for others it would appear that there is a very limited role in the following circumstances:

- critically ill patients with cardiogenic shock and multiorgan failure-good mid-term results can be obtained if secondary operation is possible; 
Percutaneous aortic valvuloplasty (PAV) and other percutaneous valve dilatation applications

- PAV for degenerative calcified aortic stenosis is of limited efficacy and provides only short term alleviation of symptoms at the cost of high procedural risk

The role of PAV should be restricted to very rare cases, if any

- Percutaneous triscuspid or polyvalvar dilatation are very seldom used

Percutaneous dilatation of bioprosthesis probably has no future

- necessity of significant emergency non-cardiac surgery in patients with severe and poorly tolerated aortic stenosis;

- cases with absolute but non-life threatening short term contraindications to surgery when a significant disability exists;

- in patients who refuse surgery.

It would appear important, however, to evaluate PAV better in rheumatic aortic stenosis because it might ultimately be an attractive application of the technique.

\section{Other applications of percutaneous valve dilatation}

Other applications of percutaneous valve dilatation are used very sparingly. The few procedures performed show that these interventions are feasible, but they are insufficient in number to allow us to evaluate results and establish indications.

At the present time it seems that indications for tricuspid valvuloplasty are rare and reserved for patients presenting a tight tricuspid stenosis, either pure or associated with mild regurgitation.

Percutaneous dilatation of bioprostheses may give rise to severe immediate complications at the level of the left heart and give poor mid term results in the tricuspid position. Therefore percutaneous dilatation may in certain rare cases be performed as a palliative for stenotic degeneration of a bioprosthesis in the tricuspid position.

\section{Conclusion}

After nearly 15 years of extensive clinical evaluation, the technique of percutaneous valvuloplasty, which for practical purposes can be summed up as percutaneous mitral commissurotomy, is now here to stay. This is because of its proven efficacy in the treatment of mitral stenosis, as a substitute for surgical commissurotomy, and a complement to valve replacement.

1. Carroll JD, Feldman T. Percutaneous mitral balloon valvotomy and the new demographics of mitral stenosis. JAMA 1993;270:1731-6.
- This articled describes the features of mitral stenosis in different parts of the world with a special emphasis on western countries.

2. Inoue K, Owaki T, Nakamura T, et al. Clinical application of transvenous mitral commissurotomy by a new balloon catheter. J Thorac Cardiovasc Surg 1984;87:394-402.

3. Vahanian A, lung B, Cormier B. Mitral valvuloplasty. In: Topol EJ, ed. Textbook of interventional cardiology.

Philadelphia: WB Saunders, 1994:821

- This chapter reviews the current techniques of percutaneous balloon commissurotomy, and the results obtained in the different subgroups.

4. Stefanadis C, Toutoutzas P. Retrograde non-transseptal mitral valvuloplasty. In: Topol EJ, ed. Textbook of interventional cardiology. Philadelphia: WB Saunders, 1994:1253.

5. Cribier A, Eltchaninoff $\mathbf{H}$, Koning $\mathbf{R}$, et al. Percutaneous mechanical mitral commissurotomy with a newly designed metallic valvulotome. Circulation 1999;99:793-9.

6. Chen CR, Cheng TO. Percutaneous balloon mitral valvuloplasty by the Inoue technique: a multicenter study of 4832 patients in China. Am Heart J 1995;129:1197-202.

7. lung $\mathbf{B}$, Cormier $\mathbf{B}$, Ducimetiere $\mathbf{P}$, et al. Immediate results of percutaneous mitral commissurotomy. Circulation 1996:94:2124-30.

- The immediate results of the PMC technique are presented and the multifactorial aspects of the predictors described.

8. Herrmann HC, Lima JAC, Feldman T, et al. Mechanisms and outcome of severe mitral regurgitation after Inoue balloon valvuloplasty. J Am Coll Cardiol

1993;27:783-9.

- This article provides an overview of the incidence, mechanisms, and predictors of traumatic mitral regurgitation.

9. lung B, Garbarz E, Michaud P, et al. Late results of clinical deterioration: frequency, anatomic findings, and predictive factors. Circulation 1999;99:3272-8. - The longest, currently available follow up following PMC is presented, together with a description of the mechanisms
of late deterioration after successful PMC and an analysis of the predictors of results.

10. Hernandez R, Bañuelos C, Alfonso F, et al. Long-term clinical and echocardiographic follow-up after percutaneous mitral valvuloplasty with the Inoue balloon. Circulation 1999;99:1580-6.

11. Shaw TRD, Mc Areavey D, Essop AR, et al. Percutaneous balloon dilatation of mitral valve in patients who were unsuitable for surgical treatment. Br Heart $J$ 1992;67:454-9.

- The results of PMC in the elderly and other patients with contraindications for surgery are reported.

12. Ben Fahrat M, Ayari M, Maatouk F. Percutaneous balloon versus surgical closed and open mitral commissurotomy. Circulation 1998;97:245-50.

- This article reports on the largest randomised comparison between PMC, closed, and open chest commissurotomy with a follow up of up to seven years.

13. lung B, Garbarz E, Doutrelant L, et al. Late results of percutaneous mitral commissurotomy for calcific mitral stenosis. Am J Cardiol 2000;85:1308-14.

- The long term results of PMC in patients with valve calcification are presented, with a special emphasis on the multifactorial nature of prediction in this subgroup.

14. Tuzcu EM, Block PC, Griffin BP, et al. Immediate and long-term outcome of percutaneous mitral valvotomy in patients 65 years and older. Circulation 1992;85:963-71.

15. lung B, Garbarz E, Michaud P, et al. Percutaneous mitral commissurotomy for restenosis after surgical mitral commissurotomy for restenosis after surgical selection. J Am Coll Cardiol 2000:35:1295-302.

16. Presbitero P, Prever SB, Brusca A. Interventional cardiology in pregnancy. Eur Heart $J$ 1996;17:182-8.

17. Cribier A, Savin T, Saoudi N, et al. Percutaneous transluminal valvuloplasty of acquired aortic stenosis in elderly patients: an alternative to valve replacement? Lancet 1986;11:63-7.

18. National Heart, Lung, and Blood Institute Balloon Registry Participants. Percutaneous balloon aortic valvuloplasty. Acute and 30-day follow-up results in 674 patients from the NHLBI balloon valvuloplasty registry. Circulation 1991;84:2383-7.

- The immediate results using the PAV technique from a large multicentre study are presented in terms of efficacy and risk

19. Otto CM, Mickel MC, Kennedy W, et al. Three-yea outcome after balloon aortic valvuloplasty: insights into prognosis of valvular aortic stenosis. Circulation 1994;89:642-50

20. Bernard Y, Etievent J, Mourand JL et al. Long-term results of percutaneous aortic valvuloplasty compared with aortic replacement in patients more than 75 years old. J Am Coll Cardiol 1992;92:1439-46. 